\title{
Fever and Psychosis as an Early Presentation of Brucella-Associated Meningoencephalitis: A Case Report
}

\author{
Mahnaz Montazeri $^{\mathrm{a}}$ Kourosh Sadeghi ${ }^{\mathrm{b}}$ Hosein Khalili $^{\mathrm{b}}$ Setareh Davoudi ${ }^{\mathrm{a}}$ \\ ${ }^{a}$ Department of Infectious Diseases, Imam Khomeini Hospital Complex, and ${ }^{b}$ Department of Clinical Pharmacy, \\ Tehran University of Medical Sciences, Tehran, Iran
}

\section{Key Words}

Brucellosis · Meningoencephalitis · Psychosis • Acyclovir • Hepatotoxicity

\section{Abstract}

Objective: To describe a case with Brucella-associated meningoencephalitis. In addition, we report drug-induced hepatotoxicity due to acyclovir. Clinical Presentation and Intervention: A young woman was admitted with fever and psychosis and neuroimaging findings indicative of meningoencephalitis. Serology was positive for Brucella. She was treated with doxycycline, rifampin, and trimethoprim-sulfamethoxazole. Conclusion: This case reminds physicians in endemic regions to consider neurobrucellosis as a differential diagnosis in patients with any unexplained neurologic symptoms or atypical psychosis. Early diagnosis and treatment of neurobrucellosis will be helpful in decreasing the sequelae of this complication.

Copyright $\odot 2013$ S. Karger AG, Basel
(C) 2013 S. Karger AG, Basel 1011-7571/13/0225-0506\$38.00/0

Karger

Open access

This is an Open Access article licensed under the terms of the Creative Commons Attribution- NonCommercial-NoDerivs 3.0 License (www.karger.com/OA-license), applicable to the online version of the article only. Distribution for non-commercial purposes only.

\section{Introduction}

Brucellosis is a zoonotic infectious disease which can be transmitted to humans via ingestion of unpasteurized dairy products, direct contact with infected animal parts or occasional inhalation of contaminated aerosols [1]. The disease is endemic in Iran. Brucella can involve multiple organs such as the central nervous system in $5-7 \%$ of cases. The most common presentation of neurobrucellosis is acute or chronic meningitis or meningoencephalitis $[1,2]$. Another rare presentation of neurobrucellosis is psychosis. Only a few cases of psychosis, as a presentation of chronic brucellosis, have been reported in the literature $[3,4]$. Here, we report another case, a young woman with brucellosis, who presented with fever and psychosis.

\section{Case Report}

A 36-year-old woman was admitted to Imam Khomeini Hospital, Tehran because of acute onset of fever and decreased level of consciousness. Her problem began 3 days prior to admission with hallucination, delusions and fever. She was single and unemployed, living in a small town, without previous history of brucellosis in

Setareh Davoud

Department of Infectious Diseases, Imam Khomeini Hospital Complex Keshavarz Boulevard

Tehran 1419733141 (Iran)

E-Maildavoudis@tums.ac.ir 

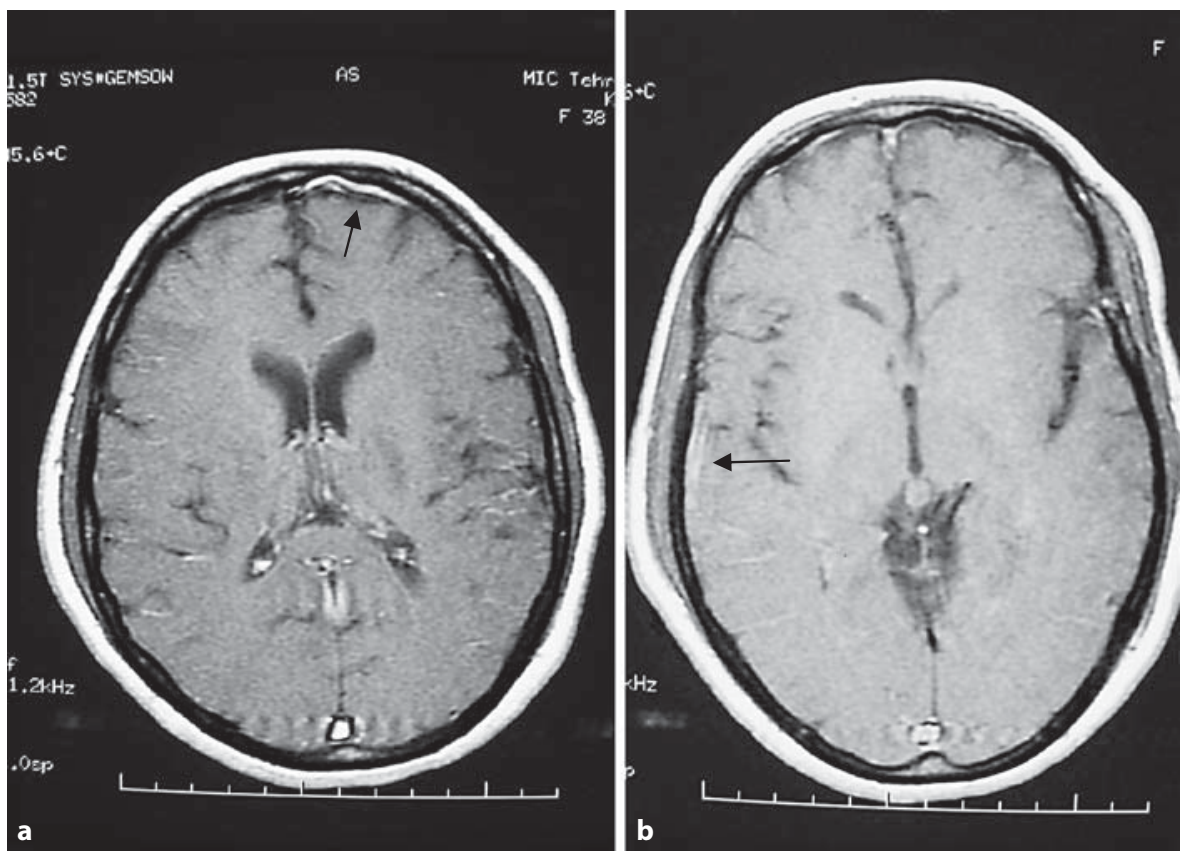

Fig. 1. The T1-weighted image after gadolinium injection shows meningeal enhancement in the left frontal lobe (a) and the right temporal lobe (b).

herself and her family. She was not receiving any medication and had no history of illicit drug use. The only positive point in her past was regular consumption of unpasteurized dairy products. On admission, she had disorientation to time and place. She was agitated and had visual hallucination and grandiose delusion. Despite her high temperature $\left(39^{\circ} \mathrm{C}\right)$ other vital signs were normal including blood pressure, pulse rate, and respiratory rate. Head and neck examination revealed nuchal rigidity. General physical examination was normal except for some abnormal neurologic findings including decreased deep tendon reflexes and forces of lower extremities at about 3/5. A lumbar puncture was done after brain computerized tomography scan did not reveal any abnormality. The results of initial tests and cerebrospinal fluid (CSF) analysis are listed in table 1 . Presence of fever, decreased level of consciousness, and bizarre behavior raised the possibility of herpes simplex virus (HSV) meningoencephalitis, hence intravenous acyclovir started pending further evaluations. Brain MRI with contrast (gadolinium) showed meningeal enhancement in left frontal and right temporal lobes (fig. 1), suggesting meningoencephalitis. During the next few days no significant change was observed in the patient's condition. On the 6th day of hospitalization she developed nausea and vomiting and laboratory tests showed significant elevation of liver enzymes (aspartate aminotransferase, AST $=1,093$, and alanine aminotransferase, ALT $=203$ ). Since our patient showed raised levels of liver function tests, we further checked for HBsAg, HCV Ab, HAV Ab (IgM), HIV Ab, EBV Ab (VCA IgM), CMV Ab (IgM), anti-mitochondrial antibody, anti-smooth muscle antibody, and liver kidney microsomal antibody; all were negative. Because of lack of clinical response to acyclovir therapy, negative CSF HSV PCR results, and possibility of drug-induced hepatotoxicity, acyclovir was discontinued after a 10-day course of therapy. At this time serology tests for brucellosis became positive including: Wright agglutination test $=1 / 40$, Coombs Wright $=1 / 80$, Brucella ELISA IgG $=146.6$ $\mathrm{U} / \mathrm{ml}$ (normal $<8 \mathrm{U} / \mathrm{ml}$ ). With probable diagnosis of neurobrucel-
Table 1. Initial laboratory tests and CSF analysis

\section{Initial tests}

$\mathrm{WBC}=5,700 / \mathrm{mm}^{3}$

$\mathrm{PMN}=69 \%$

$\mathrm{LYM}=29 \%$

$\mathrm{EOS}=1 \%$

$\mathrm{MON}=1 \%$

$\mathrm{Hb}=11.5 \mathrm{~g} / \mathrm{dl}$

$\mathrm{MCV}=82 \mathrm{fl}$

$\mathrm{MCH}=27.5 \mathrm{pg}$

$\mathrm{PLT}=208,000 / \mathrm{mm}^{3}$

$\mathrm{AST}=48(\mathrm{~N}: 0-27 \mathrm{IU} / \mathrm{l})$

$\mathrm{ALT}=27(\mathrm{~N}: 0-37 \mathrm{IU} / \mathrm{l})$

$\mathrm{ALP}=167(\mathrm{~N}:$ 80-306 IU/l)

$\mathrm{T}$ BIL $=1.1(\mathrm{~N}: 1-1.2 \mathrm{mg} / \mathrm{dl})$

$\mathrm{D} B I L=0.3(\mathrm{~N}: 0-0.2 \mathrm{mg} / \mathrm{dl})$

$\mathrm{Cr}=0.6(\mathrm{~N}: 0.6-1.1 \mathrm{mg} / \mathrm{dl})$

$\mathrm{RF}=$ neg.

ANA $=$ neg.

$\mathrm{CRP}=6 \mathrm{mg} / \mathrm{dl}$

$\mathrm{ESR}=7 \mathrm{~mm} / \mathrm{h}$

$\mathrm{BC} \times 2=$ neg.

PBS for malaria $=$ neg.

VDRL $=$ neg.

Widal test for typhoid fever $=$ neg.

\section{CSF analysis}

Color $=$ clear

Pressure $=180 \mathrm{~mm} \mathrm{H}_{2} \mathrm{O}$

Glucose $=48 \mathrm{mg} / \mathrm{dl}$

Protein $=35 \mathrm{mg} / \mathrm{dl}$

$\mathrm{LDH}=30 \mathrm{U} / 1$

$\mathrm{RBC}=70 / \mathrm{mm}^{3}$

Gram stain and culture $=$ neg.

PCR for $\mathrm{TB}=$ neg.

$\mathrm{PCR}$ for $\mathrm{HSV}=$ neg.

$\mathrm{ADA}=2 \mathrm{IU}$

$\mathrm{WBC}=10 / \mathrm{mm}^{3}(100 \%$ LYM $)$ Wright test $=$ neg.

$\mathrm{ALP}=$ Alkaline phosphatase; $\mathrm{T} \mathrm{BIL}=$ total bilirubin; $\mathrm{D} \mathrm{BIL}=$ direct bilirubin; $\mathrm{Cr}=$ creatinine; $\mathrm{ESR}=$ erythrocyte sedimentation rate; $\mathrm{BC}=$ blood culture; $\mathrm{PBS}=$ peripheral blood smear; $\mathrm{VDRL}=$ Venereal Disease Research Laboratory; $\mathrm{TB}=$ tuberculosis; $\mathrm{ADA}=$ adenosine deaminase; $\mathrm{N}=$ normal range. 
losis, triple therapy including one intravenous drug with good penetration to CSF (ceftriaxone, doxycycline and trimethoprim-sulfamethoxazole) was started. Rifampin was avoided at this time due to abnormal liver enzymes. Within a week, the patient's symptoms including fever and psychosis improved and she became completely alert without any neurological problems and regained ability to talk and eat. Five days after discontinuation of acyclovir, serum aminotransferase levels decreased $($ AST $=288 \mathrm{IU} / \mathrm{l}$ and ALT $=191$ IU/l) and subsequently 9 days after discontinuation of acyclovir, liver enzymes reached near-normal levels (AST = 54 IU/l and ALT $=63 \mathrm{IU} / \mathrm{l}$ ). Liver biopsy also showed unremarkable portal tracts and lobules. The patient was discharged with triple oral therapy (doxycycline, rifampin, trimethoprim-sulfamethoxazole). After 3 months, she became completely well. She received 6 months' treatment totally with clinical follow-ups showing no problem at 3 months and at the end of 6 months.

\section{Discussion}

The presented case was a patient with signs of meningoencephalitis due to neurobrucellosis that mimicked herpes encephalitis. The nervous system can be involved at any stage of this disease. Both central and peripheral nervous system complications have been reported [2]. Acute or chronic meningitis are the most frequent nervous system complications of brucellosis. Acute Brucella meningitis is usually characterized by sudden onset of fever, headache and nuchal rigidity, psychiatric and motor-sensory disorders $[2,5]$ as in this case.

Diagnosis of neurobrucellosis is based on: (a) clinical findings compatible with neurobrucellosis; (b) pleocytosis with predominant lymphocytes and elevated protein concentration in CSF; (c) positive results of either blood or bone marrow or CSF culture or positive serologic tests; (d) clinical improvement following antibiotic therapy against brucellosis, and (e) no other alternative diagnosis [2]. Our patient met three of them: clinical findings and serologic evidence of brucellosis, which responded well to therapy.

Psychotic manifestations of brucellosis, although uncommon, have long been recognized and reported [3, 4]. The psychiatric manifestations of neurobrucellosis are depression, amnesia, agitation, psychosis, personality disorder and euphoria. Cognitive and emotional disorders in neurobrucellosis improve by appropriate antibiotic therapy. Antipsychotic therapy is not required in this condition [5]. Neurologic presentations in the acute phase of the disease may be associated with neither pleocytosis nor increased protein levels in CSF [6]. In our case psychosis also improved with treatment of brucellosis.

Radiologic findings in neurobrucellosis are variable from completely normal to abnormal. Imaging abnor- malities can be due to an inflammatory process, white matter changes or vascular insult [7]. In this case, the imaging appearance reflected meningeal enhancement in the left frontal and right temporal lobes, which again implied the diagnosis of HSV encephalitis.

Drug-induced liver injury is a frequent adverse effect of drug therapy and can be life-threatening [8]. Nearly all medications can cause elevation of hepatic aminotransferases. A drug-induced hepatotoxicity may be considered if the increase in serum aminotransferase levels coincides with the initiation of a suspected drug [9]. Hepatotoxicity caused by drug therapy, except for rare cases, resolves by discontinuing the responsible drug. This is an important diagnostic clue for clinicians to conclude that drug therapy is responsible for liver injury [10]. In this case liver enzymes declined after eliminating acyclovir therapy. Besides, other diagnostic workups failed to show any other reason for hepatitis. It is noteworthy that hepatitis with mildly elevated transaminase level is also a common finding in brucellosis [1]. But discordance between onset of disease and time of liver enzymes increase, very high level of enzymes and AST prominence make this a less possible diagnosis. One other point that cannot be excluded in our patient is the possibility of substance abuse, which may explain elevation of liver enzymes and at least some of her clinical signs and symptoms, but both the patient and her family denied any history of substance abuse.

\section{Conclusion}

This case is to remind physicians in endemic regions to consider neurobrucellosis as a differential diagnosis in patients with any unexplained neurologic symptoms or atypical psychosis. Early diagnosis and treatment of neurobrucellosis will be helpful in decreasing the sequelae of this complication. 


\section{References}

1 Pappas G, Akritidis N, Bosilkovski M, Tsianos E: Brucellosis. N Engl J Med 2005;352: 2325-2336.

-2 Hajiabdolbaghi M, Rasoolinejad M, Jafari S, Hasibi M, Soudbakhsh A: Clinical and labaratory findings in neurobrucellosis: review of 31 cases. Arch Iran Med 2008;11:21-25.

3 Ghaffarinejad AR, Sarafzadeh F, Sedighi B, Sadeghieh T: Psychosis as an early presentation of neurobrucellosis. Iran J Med Sci 2008; 33:57-59.
4 Alapin B: Psychosomatic and somato-psychic aspects of brucellosis. J Psychosom Res 1976;20:339-350.

5 Eren S, Bayam G, Ergonula O, Celikbas A, Pazvantoglu O, Baykam N, Dokuzoguz B, Dilbaz N: Cognitive and emotional changes in neurobrucellosis. J Infect 2006;53:184-189.

-6 Karsen H, Akdeniz H, Karahocagil MK, Irmak H, Sünnetçioglu M: Toxic-febrile neurobrucellosis, clinical findings and outcome of treatment of four cases based on our experience. Scand J Infect Dis 2007;39:990-995.
7 AL-Sous MW, Bohlega S, AL-Kawi MZ, Alwataban J, Mclean DR: Neurobrucellosis: clinical and neuroimaging correlation. Am J Neuroradiol 2004;25:395-401.

8 Abboud G, Kaplowitz N: Drug-induced liver injury. Drug Saf 2007;30:277-294.

$\checkmark 9$ Pratt DS, Kaplan MM: Evaluation of abnormal liver-enzyme results in asymptomatic patients. N Engl J Med 2000;342:1266-1271.

10 Kaplowitz N: Drug-induced liver injury. Clin Infect Dis 2004;38(suppl 2):S44-S48. 